ORIGINAL RESEARCH ARTICLE

\title{
Effect of Qianjinjiu on the Contraction of Gastrocnemius muscle of Isolated Sciatic Nerve
}

\author{
Jianqing Zhu,Zhanying Qi,Chaohui Lin \\ Xiangfan Orthopedic Hospital, Hubei, China
}

\begin{abstract}
Objective: To investigate the water extract from Radix (Flemingia philippinensis Merr et Rolfe Decoction.) Effect of sciatic nerve gastrocnemius muscle Characteristics of isolated frog. Methods: The dissected lesion of sciatic nerve gastrocnemius muscle was infused with $0.9 \%$ saline, $0.125 \mathrm{~g} / \mathrm{mL}, 0.25 \mathrm{~g} / \mathrm{mL}, 0.5 \mathrm{~g} / \mathrm{mL}$ jelly extract, and the RM6240 bio-signal collector was used to measure each The infiltration depth of 5 minutes, infiltration for 10 minutes, infiltration of 15 minutes at each time point of the gastrocnemius muscle contraction threshold strength (measured at each time point 5 times) and complete single contraction curve (measured at each time point 25) Systolic rate, systolic rate, rate of contraction, and latency. Results: The systolic threshold intensity of the gastrocnemius muscle increased with the increase of the concentration of the extraction fluid, but less than the pre-treatment level $(\mathrm{P}<0.01, \mathrm{P}<0.05)$. If the concentration and time $(\mathrm{P}<0.05)$. At the same time, the systolic amplitude, systolic rate and diastolic rate increased first and then decreased with the increase of the concentration of the extract $(\mathrm{P}<0.01, \mathrm{P}<0.05)$. The latency of the sciatic nerve of the sciatic nerve was affected by the concentration of the drug in the infiltration solution, which decreased first and then increased with the increase of the drug concentration. Conclusion: The systolic characteristics of skeletal muscle are affected by the weight of the components. Low concentration of salt water extract in a short time to improve the ability of skeletal muscle contraction, treatment time is too long or too high concentration of crude drugs will make the skeletal muscle contractility weakened, and even inhibit the skeletal muscle contractility, and affect the nerve Muscle excitement signal transmission.

KEYWORDS: Qianjin pull, isolated sciatic nerve gastrocnemius, contraction characteristics
\end{abstract}

\section{The basic characteristics of a kilogram}

(Name: Flemingia philippinensis Merr. Et Rolfe), also known as: Manchuqianzhuang, piling, pony pier, a root, rat tail and drilling wind (Sichuan) [1]. Cai Yi, Liang Yan, Li Xiaoyun et al [2] on the production of three kinds of Qianzao in Guangxi had a pharmacological identification. The results are as follows: the semi-shrubs are the long cylindrical shape, slightly curved, the upper coarse and fine, occasional branches, long $20 \sim 70 \mathrm{~cm}$, diameter $1.0 \sim 1.5 \mathrm{~cm}$. Surface gray brown tan to dark brown, slightly protruding transverse lenticels and fine wrinkles tough, easy to break, slightly flat section; skin brown, broad wood, white or yellowish brown, with a clear radial Texture of gas micro, taste slightly bitter. Cork layer 2 to 4 cells, arranged closely, the cell wall is particularly thick, visible hole, cortex thinner, cells filled with reddish brown material; visible secretion chamber, containing brown secretion, phloem stenosis, secretion of cells scattered alone, Diameter of $18 \sim 30 \mu \mathrm{m}$, or a number of connected to form a large secretion chamber, the diameter of the broad woody, accounting for the surface of the 5/6; large catheter 1 to 2 poly, diameter 25 to 86 , surrounded by numerous wood fiber; , Composed of a row of flat wood parenchyma cells, containing starch granules. Root trailing radix long cylindrical shape, tapered at the thick, very few branches, length $30 \sim 70 \mathrm{~cm}$, diameter of $1.0 \sim 1.5 \mathrm{~cm}$, surface greyish brown to brown, slightly protruding long horizontal lenticels And fine wrinkles tough, easy to break, section skin dark brown, was fibrous; wide wood, light yellow and white, with subtle radial texture, micro-beanous smell, slightly sweet astringent. Cork layer for more than 10 cells, the outer side often contains dark brown, cortex narrow, and some cells containing brown matter, phloem can be seen in the secretion of cells scattered alone, diameter $18 \sim 40 \mu \mathrm{m}$, or a number of connected; fiber scattered or The diameter of $8 \sim 21 \mu \mathrm{m}$, the width of the xylem wide, accounting for $2 / 2$ of the section, a large catheter 1 to 2 poly, diameter $25 \sim 110 \mu \mathrm{m}$, surrounded by green wood; wood rays significantly from $1 \sim 8$ rows of flat wood parenchyma cells, containing calcium oxalate crystal granules or reddish brown material large leaves of the root of the root was cylindrical, more stout, and more branches, long 30 to 90 , diameter up to $6 \mathrm{~cm}$ root head slightly nodular enlargement, residual stem base rough; surface reddish brown, with small vertical lines and horizontal pimple hard, not easy to break, cross-section of the skin and wood often separated 'skin color Dark reddish brown, showing fibrous, can see the brown jelly; wood color was light brown or brown, with fine radial texture, gas micro, taste slightly Gan Xin. Cork layer for 6 to 8 cells , Tangentially extended, arranged neatly, sometimes outside

Copyright (C) 2017 -. This is an Open Access article distributed under the terms of the Creative Commons Attribution-NonCommercial 4.0 International License (http://creativecommons.org/licenses/by-nc/4.0/), permitting all non-commercial use, distribution, and reproduction in any medium, provided the original work is properly cited. 
the falling layer 'cortex Narrow, more cells containing brown material 'phloem narrow, seen in secretory cells scattered diameter of $20 \sim 32 \mathrm{~cm}$, scattered or bundled fibers; calcium oxalate crystals scattered in the xylem wide,

The diameter of $25 \sim 80 \mu \mathrm{m}$, surrounded by poly wood fiber; wood radiation is broad, from 3 to 10 cells, easy to see calcium oxalate crystal, the diameter of $10 \sim 2 \mu \mathrm{m}$ and starch granules, 5 to $20 \mu \mathrm{m}$ in diameter.

\section{Materials and reagents}

\subsection{Experimental objects and materials}

Healthy and springing normal black spotted frog, $50 \pm 5 \mathrm{~g}$, both male and female, bought in Guangxi Yulin hole market, Professor Wang Xiaoping as authentic. Raw medicine jin jiaozhu root, purchased in Guangxi Yulin City, Chinese herbal medicine market, Professor Wang Xiaoping as authentic.

\subsection{The main instrument}

RM6240 multi-channel physiological signal acquisition instrument (Chengdu Instrument Factory), precision electronic balance (Shanghai Precision Science Instrument Co., Ltd.), tension transducer, refrigerator, surgical shear, surgical tweezers, ophthalmic scissors, eye tweezers, , Fixed needle, glass minute hand, nerve shielding box, dropper, petri dish, glass minute hand, zinc copper bow, dirt cylinder, cotton thread, measuring cylinder, beaker, iron platform.

\subsection{Major drugs}

Sodium chloride (analytical grade) GB / T1266-2006 purchased from Guangdong West Long Chemical Co., Ltd., the production batch number: 1004151. American ginseng capsule $6 \mathrm{~g}(0.5 \mathrm{~g} * 12 \mathrm{pcs} / \mathrm{box}) \mathrm{Q} / \mathrm{FSTL} 0048 \mathrm{~B}$ purchased from Fujian Stellon Biological Pharmaceutical Co., Ltd., production batch number: 20140401.

\section{Experimental content and methods}

\subsection{Preparation of isolated sciatic nerve gastrocnemius experiment}

\subsubsection{Commonly used reagent preparation}

(1) Thousands of pull the extraction of water extract: the purchase of raw medicine jin with a universal crusher crushed 1 times, get thousands of pull coarse powder. In the way of decoction, with 10 times the volume of deionized water immersion 1h, heating and boiling 1h, hot filter liquid; plus 8 times the amount of water, boil 30 min, combined twice the filtrate, concentrated to $1 \mathrm{~g} / \mathrm{ml}$ spare (Diluted with deionized water to the desired concentration) [17].

(2) $0.7 \%$ frog type saline: $0.7 \mathrm{~g}$ sodium chloride dissolved in $100 \mathrm{~mL}$ deionized water that is.

(3) $0.7 \%$ American ginseng capsule solution: $3.5 \mathrm{~g}$ (ie 5) American ginseng capsule dissolved in 500mL deionized water that is.

\subsubsection{Preparation of sciatic nerve gastrocnemius}

According to the conventional method of preparation of frog sciatic nerve discharge specimens [15], destruction of the brain and spinal cord: take a frog, with gauze wrapped frogs limbs and torso, exposing the head, rinse with tap water. With the left hand holding the frog, with the index finger to suppress the front of the head so that the head before the push, thumb to press the back; right hand holding the frog needle from the occipital large hole forward piercing the cranial cavity, left and right stirring to destroy the brain tissue, foramen magnum, but do not pull out the insert after its sharp steering tube parallel to the spinal cord destroyed, when inserted into the spinal canal, frog hind legs immediately lose tension, in most cases urinary incontinence. If the spinal cord damage is complete, we can see the frog limbs relaxation, breathing disappeared. Peel: completely peel off the skin from both sides of the frog on both sides of the frog (Note: can be cut in front of the end of the caudal lobe and cloacal near the skin, so that stripping easier). And then down the frog legs, so that the head down, with surgical scissors to cut the abdominal muscles, see the spinal nerve, with rough scissors cut the spine. Right hand pinch the edge of the edge of the skin, stripped down all the hind limb skin, the specimen placed in the petri dish containing saline. Wash the hands and used the equipment and then proceed to the following steps (a) to separate the legs: with a rough scissors to cut the spine longitudinally (tail to stay on the side) and the muscles attached to the rear limbs, Cut the phalanx joint. The separated specimens were immersed in a petri dish containing physiological saline. (B) a femoral cut: take a leg, the first minute the glass along the sciatic scoliosis free abdominal portion, and the specimen fixed pin secured to the back of the ischium recognize frog plates 
clean. Sciatic nerve with the groove between the glass and the minute cycle semimembranosus biceps femoris, the thigh longitudinally separated portions of the exposed sciatic nerve until separated into popliteal nerve bifurcation. And then cut the biceps, semi-membranous and seminal muscle tendon, and around the front cut the quadriceps tendon [18]. (C) Free gastrocnemius: with a glass minute hand or tweezers separated from the gastrocnemius and Achilles tendon, and threading ligation. In the ligation of the distal with coarse scissors cut Achilles tendon, left hand line lift the gastrocnemius muscle, with the surgical cut to the surrounding tissue, but to retain the starting point of the gastrocnemius muscle and bone contact. Complete the gastrocnemius specimen: Cut the scissors from the knee around the scissors and scrape all the thigh muscles, cut the femur about $1 \mathrm{~cm}$. Discard the upper part of the femur, the retention part of the gastrocnemius muscle specimens. Place the specimen in a petri dish containing physiological saline. Test specimens with surgical tweezers to gently extract the specimen of the spine and then by $0.7 \%$ frogs saline dipped in zinc copper bow brachial nerve, such as gastrocnemius contraction, then the specimen function normal. The ligation lines on the gastrocnemius muscle were lightened and the specimens were placed in the experimental group for $0.7 \%$ frogs for 15-20 minutes.

\subsection{Skeletal muscle contraction signal acquisition experiment}

\subsubsection{Instrument connection}

The femoral head of the specimen is fixed in the bone fixation hole of the sarcolet, the nerve is placed on the sarcolemma electrode, the connecting line of the gastrocnemius tendon is connected with the tension transducer joint, the transducer output end is connected with the computer 1 channel, Sciatic nerve connected, the electrode connector and computer program stimulator output connected. Adjust the tension of the tie line, not too loose or too tight, so that the muscles naturally flattened to ensure that once the muscle contraction, can affect the tension sensor strain beam) [19]. In addition, the gastrocnemius muscle is provided with a fluid infiltration device in the double-layer glass trough to prevent the movement and drying of the specimen during the measurement, so that the measurement error occurs.

\subsubsection{Basic parameters}

Single-shot curve: single stimulus; delay: 20ms; intensity: $0.8 \mathrm{v}$; wave width: $1.0 \mathrm{~ms}$; magnification: 20 times; DC: $30 \mathrm{~Hz}$; scanning speed: $62.5 \mathrm{~ms} / \mathrm{div}$

Threshold intensity: $0.5 \mathrm{~ms}$; stimulus interval: $30 \mathrm{~ms}$; number of stimuli: 25 ; delay: $50 \mathrm{~ms}$; wave width: $1.0 \mathrm{~ms}$

\subsubsection{Grouping and processing}

The samples in 3.1.2 were divided into three groups: group were infused $5 \mathrm{~min}, 10 \mathrm{~min}, 15 \mathrm{~min}$ three times, each group were followed by $0.7 \%$ frog type saline (as negative control group), Low concentration $(0.25 \mathrm{~g} / \mathrm{ml})$, medium concentration $(0.5 \mathrm{~g} / \mathrm{ml})$, high concentration $(1 \mathrm{~g} / \mathrm{ml})$ of the extraction of water extract, $0.7 \%$ American ginseng capsule solution (as a positive control group).

\subsubsection{Identification of gastrocnemius muscle contraction}

After the instrument is connected, the skeletal muscle single shrinkage analysis, click the menu 'experimental project' in the muscle nerve test selected 'stimulus intensity and the relationship between the reaction' click, select the dialog box after the selection of single shrink experiment and set 3.2.2 basic parameters into the experimental stimulation and monitoring. After the start of the stimulus switch, when the muscle contraction, the recording threshold strength of each group measured 3 times; when the display shows the maximum shrinkage response of the complete single contraction curve (recorded a total of 25 curves), the measurement of single shrinkage of four periods: Latency, systolic, systolic and diastolic.

\subsubsection{Data records and statistical analysis}

$(\mathrm{Ms})$, systolic amplitude $(\mathrm{g})$ and systolic time $(\mathrm{ms})$ diastolic time $(\mathrm{ms})$ for each extracted single contraction curve were measured and calculated using the formula: shrinkage Rate $=$ contraction amplitude / systolic time, diastolic rate $=$ systolic amplitude / diastolic time [20]; taking the average of the third threshold intensity (v) measured values, respectively.

The experimental data were tested by origin 7.5 statistical software. The difference between the shrinkage index and the blank group was significant, and the data were expressed.

Table 1. Results 


\subsection{1 Skeletal muscle contraction signal acquisition experiment}

\section{Effects of the Extraction of Extracts on the Intensity of Gastric Muscle of Sciatic Nerve in Frog}

As shown in Table 1, the threshold intensity of each gastrocnemius muscle in the negative control group did not change significantly, and the intensity of the regional control was similar to that of the control group and the negative control group. $(\mathrm{P}<0.01, \mathrm{P}<0.05)$. The infiltration solution in group was lower than that in negative control group, and the difference was significant $(\mathrm{P}<0.01)(\mathrm{P}<0.05)$. The threshold intensity decreased first and then increased in the low, medium and high concentrations of the extract of group, and the threshold intensity decreased first and then increased with the increase of the concentration of the drug, The difference was statistically significant $(\mathrm{P}<0.01)$. Compared with the control group, the threshold strength of low, medium and high concentration of infiltration gastrocnemius muscle in group decreased, and there was significant difference between low and medium concentration $(\mathrm{P}<0.01)$, and the difference was significant at high concentration $(\mathrm{P}<0.05)$. The infiltration solution in group was low concentration and the medium concentration in the medium concentration decreased, the difference was statistically significant $(\mathrm{P}<0.05)$; the high threshold time intensity increased, the difference was statistically significant $(\mathrm{P}<(\mathrm{P}<0.01, \mathrm{P}<0.05)$. Compared with the negative control group, the control group was significantly higher than that of the control group $(\mathrm{P}<0.05)$, and the difference was statistically significant $(\mathrm{P}<(\mathrm{P}<0.01)$. There was significant difference between the two groups $(\mathrm{P}$ $<0.05)$, but there was no significant difference between the two groups $(\mathrm{P}<0.05)$, and the difference was statistically significant $(\mathrm{P}<0.05)$, Group was not statistically significant $(\mathrm{P}>0.05)$. The results showed that the threshold intensity of the gastrocnemius muscle of the sciatic nerve decreased $(\mathrm{P}<0.01)$, that was, the excitability increased. With the increase of the concentration of the extracted water, the treatment time Prolonged threshold intensity $(\mathrm{P}<0.05, \mathrm{P}>0.05)$, that is, decreased excitability, or even lower than the level before treatment.

According to records and statistical analysis

Table 2. Flemingia philippinensis Merr. Et Rolfe impact on frog sciatic gastrocnemius threshold intensity (/ v)

\begin{tabular}{|c|c|c|c|}
\hline Group & Group I & Group II & Group III \\
\hline $\begin{array}{l}\text { Negative control group } \\
\qquad(0.796 \mathrm{~g} / \mathrm{ml})\end{array}$ & $0.358 \pm 0.014$ & $0.388 \pm 0.111$ & $0.321 \pm 0.009$ \\
\hline $\begin{array}{l}\text { Low concentration } \\
\qquad(0.25 \mathrm{~g} / \mathrm{ml})\end{array}$ & $0.298 \pm 0.012^{\kappa x} \boldsymbol{\mu}$ & $0.363 \pm 0.144^{*}$ & $0.290 \pm 0.020^{\alpha \mu}$ \\
\hline $\begin{array}{l}\text { Medium concentration } \\
\qquad(0.5 \mathrm{~g} / \mathrm{ml})\end{array}$ & $0.306 \pm 0.037^{* x}$ & $0.375 \pm 0.026^{\star}$ & $0.361 \pm 0.010^{\mathrm{x}} \mathrm{H}$ \\
\hline $\begin{array}{l}\text { High concentration } \\
\qquad(1 \mathrm{~g} / \mathrm{ml})\end{array}$ & $0.318 \pm 0.093^{\cdots /}$ & $0.408 \pm 0.032^{x}$ & $0.377 \pm 0.012^{x \times}$ \\
\hline $\begin{array}{l}\text { American ginseng control } \\
\text { group }(0.7 \% \mathrm{~g} / \mathrm{ml})\end{array}$ & $0.320 \pm 0.025^{\alpha x}$ & $0.349 \pm 0.009^{*}$ & $0.299 \pm 0.006$ \\
\hline
\end{tabular}

\subsubsection{Effects of the Extraction of Extracts on the Contraction of the Sciatic Nerve of the Frog}

The results are shown in Table 2. Compared with the infiltration time of the gastrocnemius muscle, the contraction amplitude of the gastrocnemius muscle in the negative control group did not change significantly. Compared with the negative control group, the contraction amplitude of group increased first and then decreased with the increase of the concentration of the drug, and the difference was significant $(\mathrm{P}<0.05)$. The contraction amplitude of the gastrocnemius muscle $(\mathrm{P}<0.01)$. The contraction amplitude of gastrocnemius muscle at high concentration was significantly lower than that before treatment $(\mathrm{P}<0.01)$. The contraction amplitude of group increased first and then decreased with the increase of drug concentration, the difference was significant $(\mathrm{P}<0.01)$. $(\mathrm{P}>0.05)$. The contraction amplitude of gastrocnemius muscle at middle and high concentration decreased significantly, and the difference was not significant $(\mathrm{P}>0.05)$. There was no significant difference between the two groups $(\mathrm{P}>0.05)$ Significant significance 
$(\mathrm{P}<0.01)$. Compared with the control group, the contraction amplitude of groups increased with the increase of the concentration of the first and then decreased, and the contraction amplitude of the gastrocnemius muscle was significant and the difference was significant $(\mathrm{P}<0.01)$. $(\mathrm{P}<0.05)$. The contraction amplitude of group and group was significantly lower than that of negative control group $(\mathrm{P}<0.01)$, and the difference was statistically significant $(\mathrm{P}<0.05)$ the $(\mathrm{P}$ $<0.01)$, and the contraction amplitude decreased with the increase of the infiltration time $(\mathrm{P}<0.01)$. At the same time, the contraction amplitude of the gastrocnemius muscle was increased $(\mathrm{P}<0.01)$. The contraction amplitude decreased significantly compared with the pre-medication level and decreased with time $(\mathrm{P}<0.01)$.

Table 3. Flemingia philippinensis Merr. Et Rolfe impact on frog sciatic Gastrocnemius Contraction amplitude (/ g)

\begin{tabular}{|c|c|c|c|}
\hline Group & Group I & Group II & Group III \\
\hline $\begin{array}{l}\text { Negative control group } \\
(0.7 \% \mathrm{~g} / \mathrm{ml})\end{array}$ & $9.118 \pm 0.120$ & $8.192 \pm 0.180$ & $9.856 \pm 0.174$ \\
\hline $\begin{array}{c}\text { Low concentration } \\
(0.25 \mathrm{~g} / \mathrm{ml})\end{array}$ & $9.444 \pm 0.976^{\circ}$ & $9.776 \pm 0.356^{* *}$ & $9.992 \pm 0.341 ش$ \\
\hline $\begin{array}{l}\text { Medium concentration } \\
\qquad(0.5 \mathrm{~g} / \mathrm{ml})\end{array}$ & $10.378 \pm 0.273^{* *}$ & $8.328 \pm 0.161^{* *}$ & $6.318 \pm 0.0258 * *$ \\
\hline $\begin{array}{l}\text { High concentration } \\
\qquad(1 \mathrm{~g} / \mathrm{ml})\end{array}$ & $2.554 \pm 0.201^{* *}$ & $1.958 \pm 0.227^{* *}$ & $1.880 \pm 0.217 * * \mu$ \\
\hline $\begin{array}{l}\text { American ginseng control } \\
\text { group }(0.7 \% \mathrm{~g} / \mathrm{ml})\end{array}$ & $8.355 \pm 0.015^{\circ}$ & $7.255 \pm 0.015^{*}$ & $7.386 \pm 0.096^{*}$ \\
\hline
\end{tabular}

\subsubsection{Effects of the Extraction of the Extracts from the Extraction of the Extracts on the Fibular Muscle of the Sciatic Nerve in Frog}

The gastrocnemius muscle had no significant change in the rate of contraction of the gastrocnemius muscle with the increase of the infiltration time. See Table 3. Compared with the negative control group, the contraction rate of I, group increased with the increase of the concentration of the first and then decreased, the difference was significant $(\mathrm{P}<0.01)$. Compared with the control group, the contraction rate of groups increased with the increase of the concentration of the first and then decreased, the difference was significant $(\mathrm{P}<0.01)$. Compared with the negative control group, the contraction rate of gastrocnemius muscle in group and was significantly higher than that in control group $(\mathrm{P}<0.01)$. ( $\mathrm{P}$ $<0.01, \mathrm{P}<0.05)$. The concentration of gastrocnemius muscle decreased with the increase of infiltration time $(\mathrm{P}<0.01$, $\mathrm{P}<0.05)$, and the shrinkage rate of gastrocnemius muscle was decreased $(\mathrm{P}<0.01, \mathrm{P}<0.05)$, The shrinkage rate at high concentration was significantly lower than that before treatment $(\mathrm{P}<0.01)$.

Table 4. Flemingia philippinensis Merr. Et Rolfe impact on frog sciatic Gastrocnemius contraction rate relaxation rate (g $/ \mathrm{ms})()$

\begin{tabular}{|c|c|c|c|}
\hline Group & Group I & Group II & Group III \\
\hline $\begin{array}{c}\text { Negative control group } \\
(0.7 \% \mathrm{~g} / \mathrm{ml})\end{array}$ & $0.390 \pm 0.026$ & $0.368 \pm 0.020$ & $0.367 \pm 0.013$ \\
\hline $\begin{array}{l}\text { Low concentration } \\
\qquad(0.25 \mathrm{~g} / \mathrm{ml})\end{array}$ & $0.528 \pm 0.090^{* *}$ & $0.531 \pm 0.068 * * 4$ & $0.550 \pm 0.082 * *$ \\
\hline $\begin{array}{l}\text { Medium concentration } \\
\qquad(0.5 \mathrm{~g} / \mathrm{ml})\end{array}$ & $0.291 \pm 0.038^{\cdots *}$ & $0.284 \pm 0.034 * * \mu$ & $0.290 \pm 0.003^{* * *}$ \\
\hline $\begin{array}{l}\text { High concentration } \\
\qquad(1 \mathrm{~g} / \mathrm{ml})\end{array}$ & $0.264 \pm 0.040^{\cdots}$ & $0.263 \pm 0.033 * * \mu$ & $0.236 \pm 0.036 * *$ \\
\hline $\begin{array}{l}\text { American ginseng control } \\
\text { group }(0.7 \% \mathrm{~g} / \mathrm{ml})\end{array}$ & $0.459 \pm 0.031^{*}$ & $0.465 \pm 0.029^{* *}$ & $0.459 \pm 0.031 * *$ \\
\hline
\end{tabular}




\subsubsection{Effects of the Extraction of the Extracts on the Diastolic Vessel of the Sciatic Nerve in Frog}

There was no significant change in the diastolic rate of the gastrocnemius muscle with the increase of the infiltration time of the gastrocnemius muscle. See Table 4. Compared with the negative control group, the diastolic rate increased first and then decreased with the increase of the concentration of groups at low, medium and high concentration, and the diastolic rate increased significantly at the low concentration, $(\mathrm{P}<0.05)$. The difference was statistically significant $(\mathrm{P}$ $<0.01, \mathrm{P}<0.05)$. The difference was statistically significant $(\mathrm{P}<0.05)$. Compared with the control group, the diastolic rate of groups increased with the increase of the concentration of the first and then decreased, but the diastolic rate increased not very obvious, group at low concentration, the difference was significant $(\mathrm{P}<0.01)$, and the difference was statistically significant $(\mathrm{P}<0.01, \mathrm{P}<0.05)$. Compared with the negative control group, the diastolic rate of gastrocnemius muscle of group and was increased, and the difference was significant $(\mathrm{P}<0.01) .(\mathrm{P}<0.01)$. The diastolic rate of gastrocnemius muscle was decreased $(\mathrm{P}<0.01)$, and the diastolic rate was decreased $(\mathrm{P}<0.01)$ the diastolic rate at the time of concentration was significantly lower than that before treatment $(\mathrm{P}<0.01)$. See Table 4.

Table 5. Flemingia philippinensis Merr. Et Rolfe impact on frog sciatic Gastrocnemius relaxation rate (g/ms) ()

\begin{tabular}{|c|c|c|c|}
\hline Group & Group I & Group II & Group III \\
\hline $\begin{array}{l}\text { Negative control group } \\
\qquad(0.7 \% \mathrm{~g} / \mathrm{ml})\end{array}$ & $0.390 \pm 0.026$ & $0.369 \pm 0.019$ & $0.386 \pm 0.002$ \\
\hline $\begin{array}{l}\text { Low concentration } \\
\qquad(0.25 \mathrm{~g} / \mathrm{ml})\end{array}$ & $0.528 \pm 0.090^{* *}$ & $0.531 \pm 0.068 * 4$ & $0.520 \pm 0.008^{* * *}$ \\
\hline $\begin{array}{l}\text { Medium concentration } \\
\qquad(0.5 \mathrm{~g} / \mathrm{ml})\end{array}$ & $0.291 \pm 0.038^{*+\mu}$ & $0.285 \pm 0.034 * * \mu$ & $0.250 \pm 0.030 * *$ \\
\hline $\begin{array}{l}\text { High concentration } \\
\qquad(1 \mathrm{~g} / \mathrm{ml})\end{array}$ & $0.264 \pm 0.039^{* * *}$ & $0.264 \pm 0.033^{* * * 4}$ & $0.263 \pm 0.030 * * 4$ \\
\hline $\begin{array}{l}\text { American ginseng control } \\
\text { group }(0.7 \% \mathrm{~g} / \mathrm{ml})\end{array}$ & $0.465 \pm 0.029^{* *}$ & $0.465 \pm 0.029^{* *}$ & $0.479 \pm 0.033^{* *}$ \\
\hline
\end{tabular}

\subsubsection{Effects of Extraction of Water Extract on the Latent Period of Fetal Muscle of Frog in Isolated Sciatic Nerve}

The results shown in Table 5, with the infiltration of liquid increased time, before the application of sciatic nerve hilar muscle incubation period no significant change. Compared with the negative control group, the latency of low, medium and high concentration in group was lower than that before treatment, and the latency increased with the increase of concentration, the difference was statistically significant $(\mathrm{P}<0.01)(\mathrm{P}<0.01)$. There was no significant difference between the two groups $(\mathrm{P}>0.05)$, but there was no significant difference between the two groups $(\mathrm{P}>0.05)$, but there was no significant difference between the two groups $(\mathrm{P}>(\mathrm{P}<0.01)$. The latency of gastrocnemius muscle at high concentration was higher than that before treatment $(\mathrm{P}<0.05)$, and the difference was significant $(\mathrm{P}<0.01)$, and the difference was significant $(\mathrm{P}<0.05)$. Compared with the control group, the latency of the gastrocnemius muscle of and decreased with the increase of the concentration of the drug. The latency of the gastrocnemius muscle was not significant at the high concentration of group $(\mathrm{P}>0.05)$. The latency of gastrocnemius muscle was significantly larger than that of the other groups $(\mathrm{P}<0.01)$. Compared with the negative control group, the latency of gastrocnemius muscle of group and group was lower than that of negative control group, but the change was not significant (P>0.05). The latency of gastrocnemius muscle of group III was lower, the difference was statistically significant $(\mathrm{P}<0.05)$. Through the comparative analysis of the experimental data, it shows that the latent period of the sciatic nerve of the frog is affected by the concentration of the drug in the infiltration solution. The latent period of the fistula sciatic nerve gastrocnemius muscle decreased first and then increased with the increase of the concentration of jaundice, but the whole showed a decreasing trend. 
Table 6. Flemingia philippinensis Merr. Et Rolfe impact on frog sciatic Gastrocnemius incubation (/ ms) ()

\begin{tabular}{|c|c|c|c|}
\hline Group & Group I & Group II & Group III \\
\hline $\begin{array}{l}\text { Negative control group } \\
\qquad(0.7 \% \mathrm{~g} / \mathrm{ml})\end{array}$ & $12.150 \pm 0.857$ & $11.383 \pm 1.116$ & $12.473 \pm 1.582$ \\
\hline $\begin{array}{l}\text { Low concentration } \\
\qquad(0.25 \mathrm{~g} / \mathrm{ml})\end{array}$ & $8.813 \pm 0.216^{\cdots}$ & $9.185 \pm 0.254^{\cdots \mu}$ & $11.00 \pm 0.300^{\cdots 2}$ \\
\hline $\begin{array}{l}\text { Medium concentration } \\
\qquad(0.5 \mathrm{~g} / \mathrm{ml})\end{array}$ & $9.967 \pm 0.189^{-2}$ & $10.724 \pm 0.161^{*}$ & $11.02 \pm 0.526^{2 \ldots}$ \\
\hline $\begin{array}{l}\text { High concentration } \\
\qquad(1 \mathrm{~g} / \mathrm{ml})\end{array}$ & $11.46 \pm 0.361^{\cdots} \mu$ & $11.315 \pm 0.103$ & $12.716 \pm 0.125^{*}$ \\
\hline $\begin{array}{l}\text { American ginseng control } \\
\text { group }(0.7 \% \mathrm{~g} / \mathrm{ml})\end{array}$ & $12.116 \pm 1.516$ & $11.338 \pm 1.446$ & $11.733 \pm 0.517^{\circ}$ \\
\hline
\end{tabular}

\section{Discussion}

\subsection{Effects of the Effect of the Substituted Substance of 5.1 Kiln Extract on the Shrinking Characteristics of Fetal Sciatic Nerve}

In recent years, the research progress has been made in the past, involving its chemical composition, pharmacological effects, extraction technology and other aspects, the research level is increasingly deep, the research field is expanding. Studies have shown that thousands of gallbladder containing a variety of flavonoids, has been identified structures are: fleephin (flemiphilippinin) C, D. In addition, there are 5, 7, 3', 4'-tetrahydroxy-6, 8-diisoprenyl isoflavones (5, 7, 3', 4'-tetrahydroxy-6,8-diprenylisoflavone) (Flemichin) D, lupeol, $\beta$-sitosterol, and n-alkanoic acids having 22 to 30 carbon atoms. Qi Jin has a blood supplement, help Yang Road and other pharmacological effects, but whether the flavonoids for the pharmacological effects of the active material base remains to be further studied.

In addition, Mao Li et al [14] also found that thousands of pulls contained trace elements in the calcium content of the most. On the basis of normal physiological conditions, after adding non-toxic dose of $\mathrm{Ca} 2+$ at the neuromuscular junction, the increase of $\mathrm{Ca} 2+$ at the neuromuscular junction will cause more SNARE complex to bind to the Syt protein under $\mathrm{Ca} 2+$, The formation of more fusion holes to release more neurotransmitters, which makes the gastrocnemius contraction curve amplitude increases. It is proved that $\mathrm{Ca} 2+$ can enhance the excitability of the nerve, that is, it can promote the release of neurotransmitters. Neuromuscular junction, the equivalent of the central synaptosomes, the role of the same mechanism, it can be used instead of the central synaptosomes to experiment [21]. Therefore, it is of great significance to explore the mechanism of skeletal muscle contraction in the study of central nervous excitatory transmission and neuromuscular excitatory mechanism.

In this study, we used the method of water decoction to extract the crude drug composition of skeletal muscle. We used the study of skeletal muscle contraction experiment to prove the effect of Qianjin pull water on the systolic muscle of skeletal muscle, and the nerve excitatory component rich in calcium ions The effect of the extract on the neuromuscularity may be similar to that of the calcium ion. The extraction of the neurotransmitter can promote the release of the neurotransmitter at the appropriate concentration, but at the high concentration and prolonged infiltration the process may also be affected by other components of the force, making the nerve muscle excitatory assimilation stronger or alienation inhibition.

\subsection{Effects of - kg - pull - out extract on the contraction ability of frog's gastrocnemius muscle in isolated sciatic nerve}

The results of skeletal muscle contraction showed that the extraction of the skeletal muscle had significant effect on the enhancement of skeletal muscle excitability, contraction amplitude, systolic rate and diastolic rate. Concentration and time, the weight of the skeletal muscle contraction also played a significant inhibitory effect. However, the effect of Qianqian on the latent period of skeletal muscle is not obvious, indicating that the dose does not affect the end of the release of the transmitter and the excitement of the deep conduction to the process of muscle, that does not affect the speed of neuromuscular excitatory signal transmission, But the results and their mechanisms need further 
validation. Sun Hongzhao et al [20] Calcium ion studies have shown that low calcium solution significantly enhanced the contractility of toad gastrocnemius muscle, and high calcium solution significantly inhibited the contraction of toad gastrocnemius muscle. In this study, the effects of crude oil extract on neuromuscularity were studied macroscopically. It indicated that the dose of copper could affect the cell membrane potential of the neuromuscular junction, affect the nerve conduction and affect the calcium ion through the ion channel in the synaptosomes of the delivery mechanism. It can be seen in this study by measuring the contraction of the sciatic nerve gastrocnemius muscle specimens, can be a macro to reflect the amount of water extract caused by the mechanism of neurotransmitter release. In the experimental results, the high concentration of the solution significantly inhibited the contraction of the gastrocnemius muscle in vitro, which may be due to the increase in the concentration of extracellular calcium in the extracellular fluid and through some way leading to the release of intracellular sarcoplasmic reticulum $\mathrm{Ca}$ Decreased the amount of muscle contraction caused by the decline. And the low concentration of the solution can promote the contraction of the gastrocnemius muscle in vitro; the reason may be due to the temporary reduction of the gastrocnemius muscle calcium ion adjustment caused by the solution of calcium ions in the short-term receptor competition, it can also use this interpretation experiment The results of a short time in the low concentration of thousands of gallop solution has the effect of excitement. To sum up, the study shows that the daily life of the use of the role of health care or as a food, should avoid excessive use of excessive or long suffering, to avoid the impact on health.

As for the low molecular weight of the skeletal muscle in vitro excitatory effect and high concentration of insulin on the inhibition of isolated skeletal muscle micro-molecular mechanism, this study has not yet been involved, to be continued research, to do further research, Qianjin pull on the gastrocnemius muscle The real specific substances and mechanisms need to be further studied and should be controlled by quality standards, so as to provide a more reliable basis for the study of Qianjiu physiology.

\subsection{The Prospect of the Pharmacological Action}

The specific active substances and mechanisms of pharmacological action are not yet clear and need to be further studied and controlled by quality standards. This study only explored the effect of the extraction of the extract on the gastrocnemius of the sciatic nerve in the frog, and the effect was obvious, but the specific active substance and molecular mechanism of the action effect were to be further studied. There are few studies on the pharmacological efficacy of isoflavones, phenols, tannins, carbohydrates, sterols, alkaloids and amino acids contained in kilograms. Therefore, it is worthwhile to develop and utilize it and make in-depth research. In addition, in order to find the exact role of its active substances, which requires us to have a more in-depth understanding of its research methods, deeper development and so on require us to carry out further investigation and research.

\section{References}

1. Chen Peng, Weng Jianbin, Yin Hu, et al. Advances in extraction technology and pharmacological effects of the plants [J]. Shaanxi Traditional Chinese Medicine, 2013, 34 (2): 247-249.

2. Cai Yi, Liang Yan, Li Xiaoyun, et al. Guangxi three kinds of medicine and medicine identification [J]. Huaxi Pharmaceutical Journal 2012, 27 (5): 493-495.

3. Rao Wei-wen, Huang Jian-kai, Wen Zhifang, et al. Research on the quality and quality of the varieties [J]. Chinese Traditional Medicine, 1999, 30 (3): 219-221.

4. FENG Shi-xin, MA Xiao-jun, KE Fang.Preliminary Study on the Factors Affecting Seed Germination of Manchurian Seedlings [J]. Guangxi Medical Journal, 2006, 28 (6): 810-8111.

5. Chinese Journal of Pharmaceuticals, 1990, 26 (1): 42-481. [J]. Chinese Journal of Pharmaceutical Sciences, 1990,26 (1): $42-$ 481.

6. Liu Jianhua, Gao Lixin, Gao Yuqiong, et al. Studies on the Volatile Constituents of Jinzhuang [J]. Chinese Traditional Patent Medicine, 2003, 25 (6): 485-4871.

7. Guan Yanhong, Zeng Jun, Zhang Lixia. Research on the preparation of thousands of pulls and its application in Dai medicine [J]. Chinese Journal of National Medicine, 2008-10-15.

8. Chen Shuai, Wang Huizhu. Ultrasonic - assisted extraction of total flavonoids from Jinqianzhao [J]. Anhui Agricultural Sciences, 2011, 39 (36): 22311-22316.

9. Wu Zhengyi. Xinhua Materia Medica outline [M]. Shanghai: Shanghai Science and Technology Press, 1991: 1.

10. Zhu Hua, Teng Jianbei, Lin Anping, et al. Chinese Zhuangzi Chi [M]. Guangxi: Guangxi Nationalities Publishing House, 2003.

11. Chinese Journal of Gerontology, 2013; 13 (33): 3095-3097 [J] .Chinese Journal of Gerontology, Chinese Journal of Gerontology, Chinese Journal of Geriatric Dentistry.

12. Zeng Chunlan, Zhong Zhengxian, Lu Wenjie, et al. Studies on the pharmacological effects of Qianjinxian [M]. Chinese Journal of Traditional Chinese Medicine, 2011; 7 (17): 79-81.

13. Li Li, Qin Minjian, Zhang Lixia, et al. Studies on the chemical constituents and bioactivities of the plants [J]. Chinese Journal of Pharmacoepidemiology, 2009; 29 (4): 203-11. 
14. Mao Li, Tan Mingxiong, Chen Zhenfeng, et al. Determination of the content of metal elements in Guangxi and Chinese medicine by using ACP-AES method [M]. Spectroscopy and Spectral Analysis, 2009; 29 (9): 2568-2570.

15. Sun Hongzhao, Zhao Shizhen, $\mathrm{Ca} 2+$ on the systolic muscle in vitro systolic effect $[\mathrm{M}]$. Journal of Shandong Institute of Education, 2010, 1: 57-58.

16. Secher NH, Quistorff B, Dalsgaard MK.The muscles work, but that brain gets tired [J] 1Ugeskr Laeger, 2006,168 (51): 4503 $-4506$.

17. Xie Jingtian, Zhao Jing, editor. Physiological experiment (second edition) [M]. Beijing: Higher Education Press, $2002: 130$ -132, 30 -31.

18. Basic Medical Experimental Teaching Center Functional lesson plans [Z]. Baidu Library: 'Internet document resources (http: // www.wenku.baidu.c)'.

19. Skeletal muscle excitatory and contraction experiments [Z]. Biotechnology world: 'Network (http: //www.biotechwo)'.

20. Sun Hongzhao, Ai Hongbin, Qiu Jun, et al. Three kinds of anesthetics on the contraction of isolated skeletal muscle in a comparative study [M]. Journal of Shandong Normal University, 2006: 03-20.

21. Wang Hong, Li Hongfang, Chen Fang, et al. Calcium ion on the sciatic nerve gastrocnemius muscle neuromuscular neurotransmitter release effect [M]. Journal of Bethune Medical College, 2009, 3: 7. 